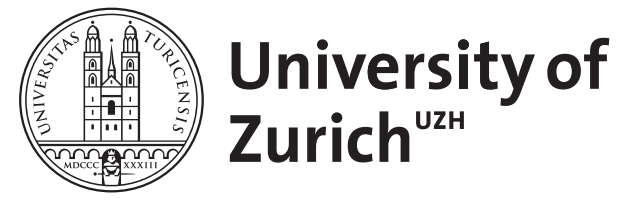

Zurich Open Repository and Archive

University of Zurich

University Library

Strickhofstrasse 39

CH-8057 Zurich

www.zora.uzh.ch

Year: 2013

\title{
Renale Regulation der Phosphathomöostase
}

Wagner, C A

DOI: https://doi.org/10.1007/s11560-013-0793-5

Posted at the Zurich Open Repository and Archive, University of Zurich

ZORA URL: https://doi.org/10.5167/uzh-156760

Journal Article

Published Version

Originally published at:

Wagner, C A (2013). Renale Regulation der Phosphathomöostase. Der Nephrologe, 8(6):523-526.

DOI: https://doi.org/10.1007/s11560-013-0793-5 
Nephrologe 2013 · 8:523-526

DOI 10.1007/s11560-013-0793-5

Online publiziert: 5. November 2013

(c) Springer-Verlag Berlin Heidelberg 2013

\section{Redaktion}

A. Kurtz, Regensburg

\section{C.A. Wagner}

Physiologisches Institut, Universität Zürich

\section{Renale Regulation der Phosphathomöostase}

aufnahme die fraktionelle Ausscheidung auf unter $5 \%$ absinkt.

\section{Bedeutung von Phosphat im Metabolismus}

Phosphat ist essenziell als Bestandteil der DNA, als Signalmolekül oder als Energieträger in ATP. Phosphatmangel verursacht Insulinresistenz, Muskelschwäche oder Hämolyse aufgrund von ATP-Mangel. Phosphat ist Bestandteil von Apatit und damit für die Stabilität von Knochen essenziell. Andererseits verursacht Überladung mit Phosphat bzw. Hyperphosphatämie, wie sie bei Patienten mit chronischer Nierenerkrankung (CKD) häufig vorkommt, Störungen wie Organverkalkungen und Arteriosklerose.

\section{Renale Resorption und Ausscheidung von Phosphat}

Beim Erwachsenen beträgt die Aufnahme von Phosphat etwa 1,4 g/Tag wovon 1,1 g im Darm absorbiert werden. Etwa $0,2 \mathrm{~g}$ werden mit Verdauungssekreten in den Stuhl ausgeschieden, 0,9 g werden über den Urin eliminiert. In der Niere wird Phosphat fast frei filtriert, rund $80 \%$ werden resorbiert und 20\% mit dem Urin ausgeschieden. Der größte Teil der Phosphatreabsorption findet im proximalen Tubulus statt, die Bedeutung und Mechanismen von Phosphatresorption im distalen Nephron sind unklar [2]. Phosphat spielt im Sammelrohr eine wichtige Rolle in der Urinansäuerung und Säureausscheidung, da Phosphat die wichtigste „titrierbare Säure“ darstellt, d. h. Protonen bindet und zur Säureausscheidung im Sammelrohr beiträgt. Bei erhöhter Phosphataufnahme kann die fraktionelle Ausscheidung von Phosphat auf deutlich über $20 \%$ ansteigen, während bei Kindern im Wachstum oder bei niedriger Phosphat-

\section{Tubuläre und molekulare Mechanismen der Phosphatresorption}

Die Resorption von Phosphat findet vor allem im initialen Anteil des proximalen Tubulus statt. Mindestens 3 verschiedene natriumabhängige Phosphattransporter (NaPi-IIa, NaPi-IIc und Pit2) vermitteln den ersten Schritt der Phosphatresorption über die luminale Bürstensaummembran. NaPi-IIa und NaPi-IIc gehören zur SLC34 (,solute carrier 34“)-Genfamilie, während Pit2 eine Isoform der SLC20Familie ist [2]. Es ist unbekannt, wie Phosphat die Zellen des proximalen Tubulus in Richtung Blut verlässt (• Abb. 1).

Funktionell unterscheiden sich NaPiIIa, NaPi-IIc und Pit2: NaPi-IIa und Na-
Pi-IIc transportieren divalentes Phosphat $\left(\mathrm{HPO}_{4}{ }^{2-}\right)$, während Pit2 monovalentes Phosphat $\left(\mathrm{H}_{2} \mathrm{PO}_{4}^{-}\right)$bevorzugt. Im proximalen Tubulus liegt der größte Teil des Phosphats in divalenter Form vor $(80 \%$ $\mathrm{HPO}_{4}{ }^{2-}$ ), damit sind $\mathrm{NaPi}$-IIa und $\mathrm{NaPi}$ IIc die wichtigsten Phosphattransporter. Der Transport eines Phosphatmoleküls ist an 3 Natriumionen bei NaPi-IIa gekoppelt, NaPi-IIc und Pit2 transportieren 2 Natriumionen pro Phosphat [2].

Die Affinität von NaPi-IIa und NaPiIIc für Phosphat ist hoch und liegt im Bereich von etwa $0,1-0,2 \mathrm{mM}$. Die maximale Transportaktivität im proximalen Tubulus, klinisch gemessen als TmP/GFR, wird vor allem durch die Zahl der aktiven Transporter in der Bürstensaummembran bestimmt (• Abb. 2). Diese ist durch den Einbau bzw. die Internalisierung der Transporterproteine bestimmt.

\begin{tabular}{|c|c|}
\hline Phosphatausscheidung wird erhöht durch: & Phosphatausscheidung wird reduziert durch: \\
\hline Hohe Phosphataufnahme & Niedrige Phosphataufnahme, Phosphatbinder \\
\hline Azidose & Alkalose \\
\hline Parathormon & Parathyroidektomie \\
\hline FGF23 & Insulin, IGF-1 \\
\hline MEPE & $\mathrm{T}_{3}$ \\
\hline \multicolumn{2}{|l|}{ sFRP4 } \\
\hline \multicolumn{2}{|l|}{ Glukokortikoide } \\
\hline \multicolumn{2}{|l|}{$\begin{array}{l}\text { Dopamin } \\
\text { Östrogene }\end{array}$} \\
\hline \multicolumn{2}{|l|}{ ANP } \\
\hline Volumenexpansion & Volumenkontraktion \\
\hline \multicolumn{2}{|l|}{ Diuretika (Azetazolamide, Thiazide) } \\
\hline Hyperkalziämie & Hypokalziämie \\
\hline \multicolumn{2}{|l|}{ Hypokaliämie } \\
\hline 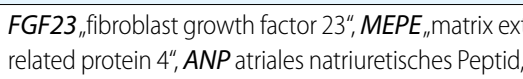 & $\begin{array}{l}\text { ellular phosphoglycoprotein", } s F R P 4{ }_{\text {,secreted frizzled }} \\
F-1 \text { "insulin-like growth factor 1", } T_{3} \text { Triiodthyronin. }\end{array}$ \\
\hline
\end{tabular}




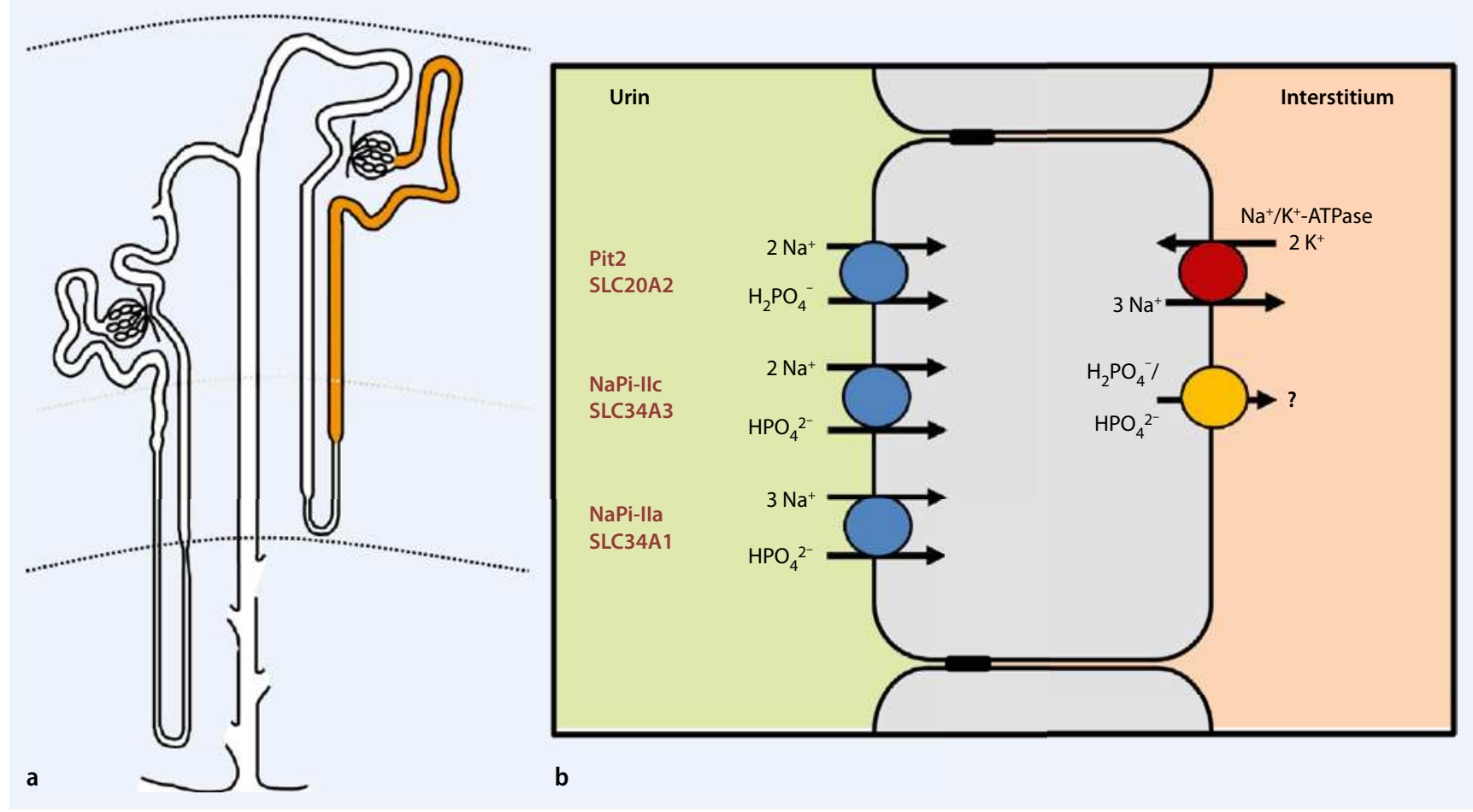

Abb. $1 \Delta$ Schematische Darstellung des Nephrons mit proximalem Tubulus ( $g e l b$, a) und einer Zelle des proximalen Tubulus (b). Die bekannten Phosphattransporter NaPi-Ila, NaPi-Ilc und Pit2 sind in der luminalen Membran lokalisiert (b)

\section{Regulation renaler Phosphattransporter}

Die renale Phosphatresorption wird von vielen Faktoren und Hormonen reguliert (• Tab. 1). Die Phosphatausscheidung wird erhöht durch Parathormon (PTH), Dopamin, Glukokortikoide und sog. Phosphatonine [2]. FGF23 („fibroblast growth factor 23“) ist das wichtigste Phosphatonin, andere Phosphatonine sind MEPE („matrix extracellular phosphoglycoprotein“) und sFRP4 („secreted frizzled related protein 4 “), wobei die Rolle von sFRP4 und MEPE beim Menschen unklar ist. IGF (,insulin-like growth factor")-1, Insulin oder Schilddrüsenhormon $\left(\mathrm{T}_{3}\right)$ stimulieren die renale Phosphatreabsorption [2]. Der Säure-Basen-Status beeinflusst die proximalen Phosphattransporter durch direkte Hemmung der NaPi-IIa- und NaPi-IIc-Transporter durch Protonen, sodass Phosphat bei Azidose vermehrt und bei Alkalose verringert ausgeschieden wird. Die Regulation durch die Phosphataufnahme mit der Nahrung ist komplexer: Phosphataufnahme stimuliert die PTH- und FGF23-Sekretion, wobei PTH nach wenigen Minuten ansteigt, während FGF23 erst mit 4-6 h Verzögerung reagiert und damit wohl weniger zur akuten Regulation beiträgt [3]. Im Tiermodell adaptiert die Niere auch in Abwesenheit von PTH innerhalb von 1-2 h an die Phosphataufnahme. Dies und die Tatsache, dass Zellkulturen von proximalen Tubuluszellen in vitro ihre Phosphattransportkapazität an die Phosphatkonzentration im Medium adaptieren, unterstützt die Hypothese, dass renale und andere Zellen die Fähigkeit besitzen, Phosphat zu messen und darauf zu reagieren.

Die Wirkung von $1,25(\mathrm{OH})_{2}$-Vitamin $\mathrm{D}_{3}$ (Cholecalciferol) auf die Phosphatausscheidung ist differenziert. Phosphatmangel erhöht die renale Synthese des aktiven $1,25(\mathrm{OH})_{2}$-Vitamin $\mathrm{D}_{3}$, welches den intestinalen und den renalen Phosphattransport stimuliert. Zumindest bei Mäusen adaptieren aber Darm und Niere auch in Abwesenheit von Vitamin $\mathrm{D}_{3}$ und seines Rezeptors [2]. Akute Gabe von Vitamin $\mathrm{D}_{3}$ in nichtphosphatdepletierten Tieren führt dagegen zur Hemmung der renalen Phosphatresorption. Paradoxerweise stimuliert Cholecalciferol einerseits die renale Phosphatresorption während einer Phosphatdepletion oder bei chronisch erhöhten Hor- monspiegeln (Tage bis Wochen), andererseits hemmt die kurzfristige Gabe oder Ausschüttung (Stunden bis wenige Tage) von Cholecalciferol die Phosphatresorption und wirkt phosphaturisch. Die Interaktionen zwischen Cholecalciferol, PTH und FGF23 und der direkte Einfluss des Phosphatstatus erklären eventuell diese Zeitabhängigkeit.

\section{Zelluläre Mechanismen der Regulation der renalen Phosphatresorption}

Die Resorption von Phosphat wird durch die Anzahl von Transportern in der Bürstensaummembran des proximalen $\mathrm{Tu}$ bulus bestimmt. Die Anzahl von Transportern wird durch den Einbau bzw. die Internalisierung von Transportproteinen reguliert. Direkte Modulation der Transportaktivität von membranständigen Transportern wurde bislang mit Ausnahme der Kaliumdepletion nicht gefunden.

Die Mechanismen des regulierten Einbaus von Transportmolekülen in die Bürstensaummembran ist wenig erforscht, die der Internalisierung wurden intensiver untersucht. Akute Gabe von Parathormon oder Fütterung mit phosphatrei- 

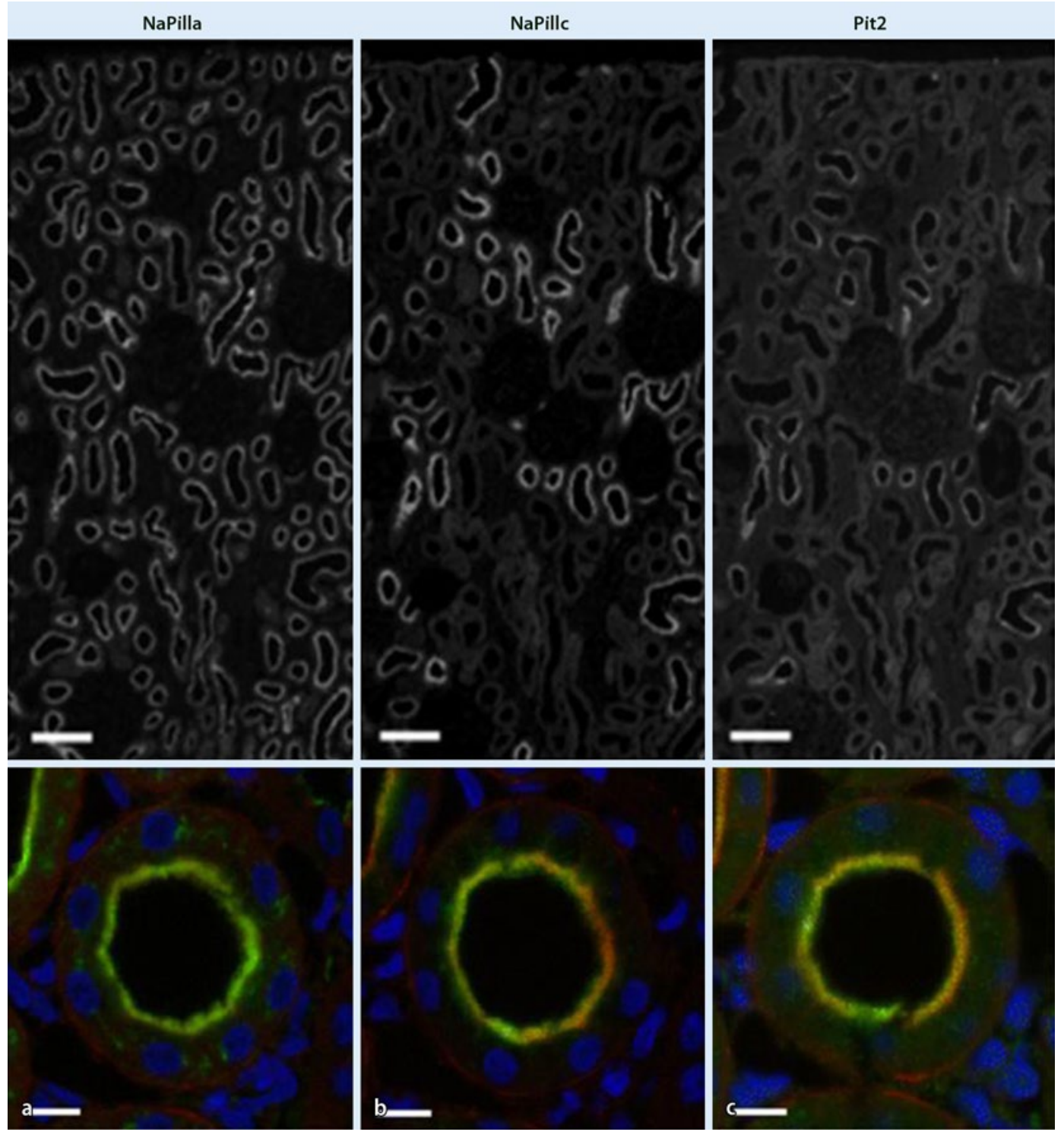

Abb. $2<$ Immunhistochemisch sind alle 3 Transporter (NaPilla, NaPillc, Pit2; grün) in der Bürstensaummembran (rot) des proximalen Tubulus nachweisbar (gelb bei Kolokalisierung). (Mod. nach [6])

cher Nahrung führt bei Nagern innerhalb von Minuten zur Internalisierung von NaPi-IIa-Transportern und zur Degradation in Lysosomen [1]. NaPi-IIc- und Pit2-Transporter werden erst nach Stunden internalisiert, wobei NaPi-IIc rezykliert wird [2]. Parathormon induziert die NaPi-IIa-Internalisierung über PTH1Rezeptoren über Signale, die die Proteinkinasen A und C sowie MAP-Kinasen involvieren [2]. Diese Kinasen führen zur Phosphorylierung von NHERF1 („Na ${ }^{+}$/ $\mathrm{H}^{+}$-exchanger regulating factor $\left.1^{\prime \prime}\right)$, der $\mathrm{NaPi}$-IIa in der Bürstensaummembran stabilisiert und nach der Phosphorylierung die Bindung an NaPi-IIa löst [2].

Eine Reihe weiterer Kinasen wie GSK3, AKT2/Proteinkinase B, JAK2, OSR1,
SPAK und SGK3 spielt für die Regulation von NaPi-IIa eine wichtige Rolle.

\section{Wechselspiel phosphat- regulierender Hormone}

Die Interaktion der wichtigsten Hormone, die den Phosphathaushalt regulieren, ist in den letzten Jahren Gegenstand intensiver Forschung und nicht abschließend verstanden; drei Hormone spielen eine herausgehobene Rolle, und zwar Parathormon, FGF23, und Vitamin $\mathrm{D}_{3}$.

Die Bildung und die Sekretion von PTH in den Nebenschilddrüsen sind unter der Kontrolle des Kalziumrezeptors, der die Konzentration des ionisierten Kalziums in der Extrazellulärflüssig- keit misst. Die Zellen der Nebenschilddrüse sind auch in der Lage, Veränderungen der extrazellulären Phosphatkonzentration unabhängig von Kalzium zu messen und an die Sekretion von PTH zu koppeln [3], d. h. ein Anstieg der Plasmaphosphatkonztration kann direkt die PTH-Sekretion stimulieren. PTH hemmt innerhalb weniger Minuten in der Niere die Phosphatresorption und trägt so zur Normalisierung der Plasmaphosphatkonzentration bei.

PTH interagiert sowohl mit Vitamin $\mathrm{D}_{3}$ als auch mit FGF23. In der Niere stimuliert PTH die Synthese von $1,25(\mathrm{OH})_{2}{ }^{-}$ Vitamin $\mathrm{D}_{3}$ durch erhöhte Expression der 1-Alpha-Hydroxylase im proximalen $\mathrm{Tu}$ bulus. Im Knochen stimuliert PTH die 


\section{Hier steht eine Anzeige.}

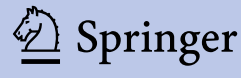

Synthese und die Freisetzung von FGF23, während $1,25(\mathrm{OH})_{2}-\mathrm{D}_{3}$ und FGF23 die Synthese und Sekretion von PTH hemmen. Erhöhtes Phosphat wirkt auf diesen Regulationskreis auch direkt, und zwar durch höhere Freisetzung von PTH, Stimulation der FGF23-Bildung im Knochen und durch Unterdrücken der Synthese von $1,25(\mathrm{OH})_{2}$-Vitamin $\mathrm{D}_{3}$ und Beschleunigung seines Abbaus über die 24-Hydroxylase.

Bei Patienten mit CKD ist dieser Regulationskreis gestört: Die Synthese von Vitamin $\mathrm{D}_{3}$ in der Niere ist verringert, die Nebenschilddrüse sezerniert vermehrt PTH, und FGF23 wird vermehrt gebildet. Studien in kleinen Patientenkollektiven deuten darauf hin, dass sich der Anstieg von FGF23 schon vor einem messbaren Anstieg der Blutspiegel von Phosphat und PTH vollzieht, jedoch erst nachdem die Vitamin- $\mathrm{D}_{3}$-Spiegel abgefallen sind. Die Konzentration des Korezeptors von FGF23, Klotho, in seiner löslichen Form fällt vor dem Anstieg von FGF23 ab. Inwiefern der Anstieg von FGF23 eine Kompensation der verringerten Klotho-Spiegel oder der drohenden systemischen Überladung mit Phopshat ist, bleibt zu klären. Patienten mit autosomal-dominanter polyzystischer Nierenerkrankung (ADPKD) zeigen bei eGFR (geschätzte glomeruläre Filtrationsrate)-Werten über $80 \mathrm{ml} / \mathrm{min}$ schon deutlich erhöhte FGF23-Werte [5]. Im Tiermodell produziert auch die Niere FGF23 (unveröffentlichte Daten; D. Spichtig, P. Jaeger, A. Serra und C.A. Wagner).

\section{Ausblick}

Die renale Phosphatausscheidung ist kritisch für die Phosphathomöostase und wird durch eine Vielzahl von Faktoren reguliert. Die Integration dieser Signale ist noch nicht komplett verstanden, und einiges deutet darauf hin, dass auch nicht alle Signalmolekule identifziert sind. Ob und wie die Niere (und andere Organe) auch direkt die Aufnahme von Phosphat, die Füllung des körpereigenen Phosphatspeichers oder die Höhe des Phosphatspiegel messen kann, ist unklar. In diesem Zusammenhang ist die Frage wichtig, warum FGF23 bei zunehmender Verschlechterung der Nierenfunktion an- steigt. Tierversuche zur Neutralisierung der hohen FGF23-Spiegel zeigen kontroverse Ergebnisse bezüglich Überleben der Tiere und Verbesserung der Nierenfunktion [4]. Offen ist auch die molekulare Identifikation der basolateralen Phosphattransporter in Niere und Darm. Zumindest im Darm scheint ein Großteil der Phosphataufnahme auch transporterunabhängig über parazelluläre Wege vermittelt zu werden - auch diese Mechanismen sind unverstanden.

\section{Korrespondenzadresse

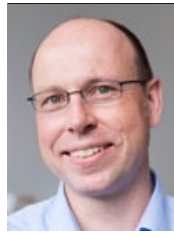 \\ Prof. Dr. C.A. Wagner \\ Physiologisches Institut, Universität Zürich Winterthurerstr. 190, CH-8057 Zürich, Schweiz Wagnerca@access.uzh.ch}

Danksagung. Die Arbeiten unserer Gruppe werden durch den Schweizerischen Nationalfonds im Rahmen des National Center of Competence in Research (NCCR), Kidney.CH unterstützt. Darüber hinaus wird unsere Forschung durch die Michael J. Fox Foundation, die Gottfried und Julia Bangerter-Rhyner-Stiftung für medizinische Forschung und die Stiftung der SwissLife unterstützt.

\section{Einhaltung ethischer Richtlinien}

Interessenkonflikt. C.A. Wagner gibt an, dass kein Interessenkonflikt besteht.

Dieser Beitrag beinhaltet keine Studien an Menschen oder Tieren.

\section{Literatur}

1. Bacic D, Lehir M, Biber J et al (2006) The renal $\mathrm{Na}+$ / phosphate cotransporter NaPi-lla is internalized via the receptor-mediated endocytic route in response to parathyroid hormone. Kidney Int 69:495-503

2. Biber J, Hernando N, Forster I (2013) Phosphate transporters and their function. Annu Rev Physiol 75:535-550

3. Bourgeois S, Capuano P, Stange G et al (2013) The phosphate transporter NaPi-lla determines the rapid renal adaptation to dietary phosphate intake in mouse irrespective of persistently high FGF23 levels. Pflugers Arch [Epub ahead of print]

4. Ketteler M, Biggar PH, Liangos O (2013) FGF23 antagonism: the thin line between adaptation and maladaptation in chronic kidney disease. Nephrol Dial Transplant 28:821-825

5. Pavik I, Jaeger P, Ebner L et al (2012) Soluble klotho and autosomal dominant polycystic kidney disease. Clin J Am Soc Nephrol 7:248-257

6. Picard N, Capuano P, Stange G et al (2010) Acute parathyroid hormone differentially regulates renal brush border membrane phosphate cotransporters. Pflugers Arch 460(3):677-687 